\title{
Equivalence between the planar Dirac oscillator and a spin-1/2 fermion embedded in a transverse homogeneous magnetic field
}

\author{
Antonio Soares de Castro*1] \\ ${ }^{1}$ Universidade Estadual Paulista "Júlio de Mesquita Filho", Departamento de Física e Química, Guaratinguetá, SP, Brasil
}

Received on April 22, 2019; Accepted on June 4, 2019.

\begin{abstract}
It is shown that a spin- $1 / 2$ fermion coupled to the axially symmetric electromagnetic vector potential has the same matrix structure as that one for the planar Dirac oscillator. In particular, the planar Dirac oscillator can be interpreted as a charged particle minimally coupled to a transverse homogeneous magnetic field.
\end{abstract}

Keywords: Dirac oscillator, relativistic planar motion, transverse homogeneous magnetic field.

\section{Introduction}

The Dirac oscillator is an exactly solvable model consisting in a nonminimal coupling prescription in the Dirac equation with the resulting equation linear in both momentum and position operators [1]. Recently, much interest has been generated on the planar Dirac oscillator. In particular, has been investigated the bound-state spectrum and its degeneracy [2]- [3], and applications to quantum optical phenomena $[4]-[5]$. Addition of a transverse uniform magnetic field has triggered further investigations related to the Aharonov-Bohm [6]- 7] and Aharonov-Bohm-Coulomb effects 8], coherent states [9], optical models [10- 12] and graphene [7], 13]- [18. It should be mentioned that the authors of Refs. 10 and [11 have correctly recognized that the planar Dirac oscillator immersed in a transverse homogeneous magnetic field can be mapped on a pure planar Dirac oscillator.

The present work shows in a simple way the exact equivalence between the planar Dirac oscillator and the problem of a charged particle minimally coupled to a transverse magnetic field. Beyond a content interesting and easy to deal with by graduate students in Physics, this result is of great importance to help to clear up disagreements relating to the bound states and its degeneracy, to assist the mapping of the planar Dirac oscillator onto quantum optical models and graphene, and to assert the appropriate chirality of the system needed to look into the critical magnetic field and the possible chirality quantum phase transition relevant to applications in quantum optical models and graphene.

*Correspondence email address: antonio.castro@unesp.br.

\section{Dirac equation with an axially symmetric electromagnetic vector potential}

In the Minkowski space-time, the behavior of a spin$1 / 2$ fermion of mass $m$ and electric charge $q$ interacting with a stationary magnetic field is governed by the Dirac equation

$$
i \frac{\partial \Psi}{\partial t}=H \Psi=[\vec{\alpha} \cdot(\vec{p}-q \vec{A})+\beta m] \Psi,
$$

with $\vec{p}=-i \vec{\nabla}$ (in natural units $\hbar=c=1$ ). Here we have used the minimal coupling prescription

$$
\vec{p} \rightarrow \vec{p}-q \vec{A}
$$

The magnetic field is described by $\vec{B}=\vec{\nabla} \times \vec{A}$, and the matrices $\vec{\alpha}$ and $\beta$ can be represented as

$$
\vec{\alpha}=\left(\begin{array}{cc}
0 & \vec{\sigma} \\
\vec{\sigma} & 0
\end{array}\right), \quad \beta=\left(\begin{array}{cc}
I_{2 \times 2} & 0 \\
0 & -I_{2 \times 2}
\end{array}\right) .
$$

where $I_{2 \times 2}$ is the $2 \times 2$ unit matrix and $\vec{\sigma}=\left(\sigma_{1}, \sigma_{2}, \sigma_{3}\right)$. The spinor $\Psi$ has four components and the $2 \times 2$ Pauli matrices obey the fundamental relation

$$
\sigma_{i} \sigma_{j}=\delta_{i j} I_{2 \times 2}+i \sum_{k=1}^{3} \varepsilon_{i j k} \sigma_{k},
$$

where $\delta_{i j}$ is the Kronecker delta and $\varepsilon_{i j k}$ is the LeviCivita symbol. In cylindrical coordinates $\left(\rho, \varphi, x_{3}\right)$ one has $\rho=|\vec{\rho}|=\sqrt{x_{1}^{2}+x_{2}^{2}}$ and $\varphi=\arctan \left(x_{2} / x_{1}\right)$ with coordinate unit vectors

$$
\begin{aligned}
\widehat{\rho} & =\cos \varphi \widehat{e}_{1}+\sin \varphi \widehat{e}_{2} \\
\widehat{\varphi} & =-\sin \varphi \widehat{e}_{1}+\cos \varphi \widehat{e}_{2} \\
\widehat{e}_{3} & =\widehat{e}_{3},
\end{aligned}
$$


and

$$
\vec{\nabla}=\widehat{\rho} \frac{\partial}{\partial \rho}+\frac{\widehat{\varphi}}{\rho} \frac{\partial}{\partial \varphi}+\widehat{e}_{3} \frac{\partial}{\partial x_{3}} .
$$

The axially symmetric electromagnetic vector potential

$$
\vec{A}=A_{\varphi}(\rho) \widehat{\varphi}
$$

gives a transverse magnetic field

$$
\vec{B}=B(\rho) \widehat{e}_{3},
$$

with

$$
B(\rho)=\frac{1}{\rho} \frac{d\left[\rho A_{\varphi}(\rho)\right]}{d \rho} .
$$

Use of the axially symmetric electromagnetic vector potential allows the Hamiltonian to be written as

$$
H=-i \alpha_{\rho} \frac{\partial}{\partial \rho}-i \alpha_{\varphi}\left(\frac{1}{\rho} \frac{\partial}{\partial \varphi}-i q A_{\varphi}\right)-i \alpha_{3} \frac{\partial}{\partial x_{3}}+\beta m
$$

where $\alpha_{\rho}=\vec{\alpha} \cdot \widehat{\rho}$ and $\alpha_{\varphi}=\vec{\alpha} \cdot \widehat{\varphi}$, with

$$
\begin{gathered}
\sigma_{\rho}=\vec{\sigma} \cdot \widehat{\rho}=\left(\begin{array}{cc}
0 & e^{-i \varphi} \\
e^{+i \varphi} & 0
\end{array}\right), \\
\sigma_{\varphi}=\vec{\sigma} \cdot \widehat{\varphi}=\left(\begin{array}{cc}
0 & -e^{-i \varphi} \\
e^{+i \varphi} & 0
\end{array}\right) .
\end{gathered}
$$

\section{The exact equivalence with the planar Dirac oscillator}

It is remarkable that $A_{\varphi}(\rho)$ in the second term of the Hamiltonian (multiplied by $\alpha_{\varphi}$ ) expressed by $(10 p$ can be moved to the first term (multiplied by $\alpha_{\rho}$ ). This happens because $\sigma_{\varphi}=i \sigma_{\rho} \sigma_{3}$ in such a way that $\alpha_{\varphi}=i \alpha_{\rho} \Sigma_{3}$. Here,

$$
\Sigma_{3}=\left(\begin{array}{cc}
\sigma_{3} & 0 \\
0 & \sigma_{3}
\end{array}\right)
$$

Therefore, the Hamiltonian expressed by 10 can also be written as

$H=-i \alpha_{\rho}\left(\frac{\partial}{\partial \rho}+q \Sigma_{3} A_{\varphi}\right)-i \alpha_{\varphi} \frac{1}{\rho} \frac{\partial}{\partial \varphi}-i \alpha_{3} \frac{\partial}{\partial x_{3}}+\beta m$,

or equivalently,

$$
H=\vec{\alpha} \cdot\left(\vec{p}-i q \Sigma_{3} A_{\varphi} \widehat{\rho}\right)+\beta m
$$

This is an extraordinary result. The Hamiltonian expressed by (14) has the same matrix structure as that one of the planar Dirac oscillator but with a more general radial potential function due to the more general form for the axially symmetric electromagnetic vector potential $A_{\varphi}(\rho)$. The problem of a charged particle minimally coupled to a transverse magnetic field and the planar Dirac oscillator become indistinguishable when the transverse magnetic field is uniform $\left(A_{\varphi}=B \rho / 2\right)$ and the cyclotron frequency $|q| B /(2 m)$ is identified with the frequency of the Dirac oscillator.

\section{Final remarks}

For short, we showed that the planar Dirac oscillator for an electrically charged particle can be interpreted as the problem describing a spin- $1 / 2$ fermion minimally coupled to a transverse homogeneous magnetic field. Hence, their bound-state spectra and degeneracies are undoubtedly the same. Applications of the planar Dirac oscillator to describe quantum optical phenomena are also equivalent to applications of a transverse homogeneous magnetic field. Addition of a transverse uniform magnetic field to a planar Dirac oscillator clearly appears to be redundant.

\section{Acknowledgement}

This work was supported in part by means of funds provided by CNPq (grant 304743/2015-1).

\section{References}

[1] M. Moshinsky and A. Szczepaniak, J. Phys. A 22, L817 (1989).

[2] V.M. Villalba, Phys. Rev. A 49, 586 (1994).

[3] F.M. Andrade and E.O. Silva, Eur. Phys. Lett. 108, 30003 (2014) .

[4] A. Bermudez, M.A. Martin-Delgado and E. Solano, Phys. Rev. A 76, 041801(R) (2007).

[5] A. Bermudez, M.A. Martin-Delgado and A. Luis, Phys. Rev. A 77, 033832 (2008).

[6] N. Ferkous and A. Bounames, Phys. Lett. A 325, 21 (2004).

[7] J. Amaro Neto, M.J. Bueno and C. Furtado, Ann. Physics 373, 273 (2016).

[8] R.R.S. Oliveira, R.V. Maluf and C.A.S. Almeida, Ann. Phys. 400, 1 (2018).

[9] D. Ojeda-Guillén, R.D. Mota and V.D. Granados, Comm. Theor. Phys. 63, 271 (2015).

[10] B.P. Mandal and S. Verma, Phys. Lett. A 374, 1021 (2010).

[11] B.P. Mandal and S.K. Rai, Phys. Lett. A 376, 2467 (2012).

[12] Y.L. Hou, Q. Wang and Z.W. Long, J. Jing, Int. J. Theor. Phys. 54, 1506 (2015).

[13] C. Quimbay and P. Strange, arXiv:1311.2021 (2013).

[14] C. Quimbay and P. Strange, arXiv:1312.5251 (2013)

[15] M.J. Bueno, J.L. de Melo, C. Furtado and A.M.M. Carvalho, Eur. Phys. J. Plus 129, 201 (2014).

[16] A. Boumali, Phys. Scr. 90, 045702 (2015).

[17] A. Belouad, A. Jellal and Y. Zahidi, Phys. Lett. A 380 773 (2016).

[18] N. Hatami and M.R. Setare, Phys. Lett. A 380, 3469 (2016). 